\title{
Effect of temperature on the nucleation and growth of precious metal nanocrystals
}

\author{
Anaïs Pitto-Barry* and Nicolas P. E. Barry*
}

\begin{abstract}
Understanding the effect of physical parameters (e.g. temperature) on crystallisation dynamics is of paramount importance for the synthesis of nanocrystals of well-defined sizes and geometries. However, imaging nucleation and growth is an experimental challenge owing to the resolution required and the kinetics involved. Here, using an aberration-corrected transmission electron microscope, we report the fabrication of precious metal nanocrystals from nuclei and the identification of the dynamics of their nucleation at three different temperatures $\left(20,50\right.$, and $\left.100{ }^{\circ} \mathrm{C}\right)$. A fast, and apparently linear, acceleration of the rate of nucleation is observed against increasing temperature $(78.8,117.7$, and 176.5 $\mathrm{pm} / \mathrm{min}$, respectively). This work appears to be the first direct observation of the effect of temperature on the nucleation and growth of metal nanocrystals.
\end{abstract}

Nanocrystals have the potential to be transformative in catalysis, electronics, and healthcar ${ }^{[1]}$ because their sizes and dimensions have an influence on their properties at the micro and macro scales. ${ }^{[2]}$ Achieving direct control over the parameters dictating their growth is therefore highly desirable. ${ }^{[3]}$ However, understanding the formation of such nanocrystals is extremely challenging owing to the timeframe on which initial events take place, the atomic scale, and the non-equilibrium conditions. Time-resolved studies at individual atomic resolution (e.g. dynamics of $\mathrm{Si}_{6}$ clusters, ${ }^{[4]}$ metal atoms trapped in graphene, ${ }^{[5]}$ random walks of defects in graphene, ${ }^{[6]}$ or visualisation of clusters of gold atoms ${ }^{[7]}$ ) have been reported but little is known about the early stages of nanocrystallisation.

Recent progresses in microscopy have allowed for the development of the chemTEM approach. ${ }^{[8]}$ This approach uses both capabilities of modern high-resolution transmission electron microscopy (TEM; sub-angstrom imaging probe and electron beam as a tunable source of energy) to induce chemical transformations at the atomic level, with concomitant imaging of such transformations in real time and in real space. [3d]

In 2014, we developed a new methodology based on this chemTEM framework, which allows the observation of nanocrystals growth. ${ }^{[9]}$ Taking advantage of the dual capabilities $^{[10]}$ of a TEM, we irradiate metallated micelles with a powerful electron beam. Upon irradiation, the metallated micelles decompose and individual metal atoms migrate to form nanocrystals on a self-supporting multi-doped graphitic surface. This methodology has allowed us to image and capture the

Dr A. Pitto-Barry, Dr N. P. E. Barry

School of Chemistry and Biosciences

University of Bradford

Bradford BD7 1DP, UK.

E-mails: a.pitto-barry@bradford.ac.uk; n.barry@bradford.ac.uk

Supporting information for this article is given via a link at the end of the document. growth and nucleation rates of ruthenium, osmium, iridium, and gold nanocrystals. ${ }^{[11]}$ By modifying the doping of the graphitic surface, we showed that hetero-atoms can act as trapping sites for individual metal atoms, by slowing their motions on the surface. ${ }^{[12]}$ These studies have allowed us to demonstrate that the nucleation rate-dependency is related to both the nature of the metal and the doping atoms within the supporting surface. In this study, we report the fabrication of osmium nanocrystals on silicon nitride membranes by electron-beam irradiation in the TEM chamber of an environmental tomographic transmission electron microscope (ETEM), used as an aberration-corrected TEM, at three different temperatures $\left(20,50\right.$, and $\left.100^{\circ} \mathrm{C}\right)$. The nucleation rates are studied for each temperature and the energy barrier of the observed thermally activated process is estimated. This energy barrier is compared with the kinetic energy transferred from the e-beam to an osmium, carbon, boron, or sulfur atom. An attempt at determining the effect of temperature on the crystallographic phases is also made, thereby factoring the effect of temperature in our understanding of the nucleation stage of the nanocrystallisation processes.

OsMs block copolymer micelles containing 16-electron dithiocarborane complexes of Os(II) were prepared according to our previous report, ${ }^{[13]}$ deposited on silicon nitride membrane $(1 \mathrm{mg} / \mathrm{mL})$ and irradiated with the electron beam of an environmental FEI-TITAN tomographic transmission electron microscope (used as an aberration-corrected High Resolution TEM operated at $300 \mathrm{keV} ; 1.9 \mathrm{pA} \cdot \mathrm{cm}^{-2} ; 7.6 \times 10^{7}$ electrons $\cdot \mathrm{nm}^{-2} \cdot \mathrm{s}$ 1 , Figure $1 \mathrm{a}$ ) at $20^{\circ} \mathrm{C}$. Within minutes, structural changes within the polymer matrix were observed upon irradiation with the formation of nanocrystals (Figure 1a; Figure 1c shows the difference of contrast between Os and the background).

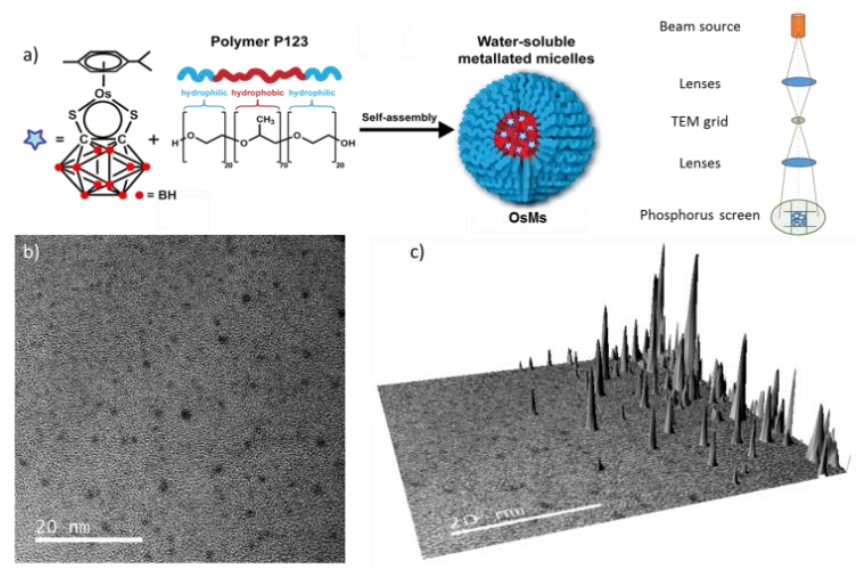

Figure 1. a) Self-assembly of block copolymer micelles containing encapsulated Os carborane complexes and electron beam irradiation of the metallated micelles; b) HRTEM image showing the degradation of the metallated micelles and production of a graphitic surface with concomitant formation of metal nanocrystals; c) 3D projection of the same image. 
The same experiments were carried out at $50^{\circ} \mathrm{C}$ and $100{ }^{\circ} \mathrm{C}$, leading to the similar in situ generation of crystalline precious metal nanoclusters (see ESI: "Sample preparation" tab in the spreadsheet). The observation that nanocrystals can be formed on the TEM silicon nitride window generalises our method previously described on TEM copper grids with lacey carbon. Furthermore, the increase of temperature does not impair the fabrication of nanocrystals. Encouraged by these results, we then imaged the early steps of nuclei aggregation for each temperature (Figure 2).

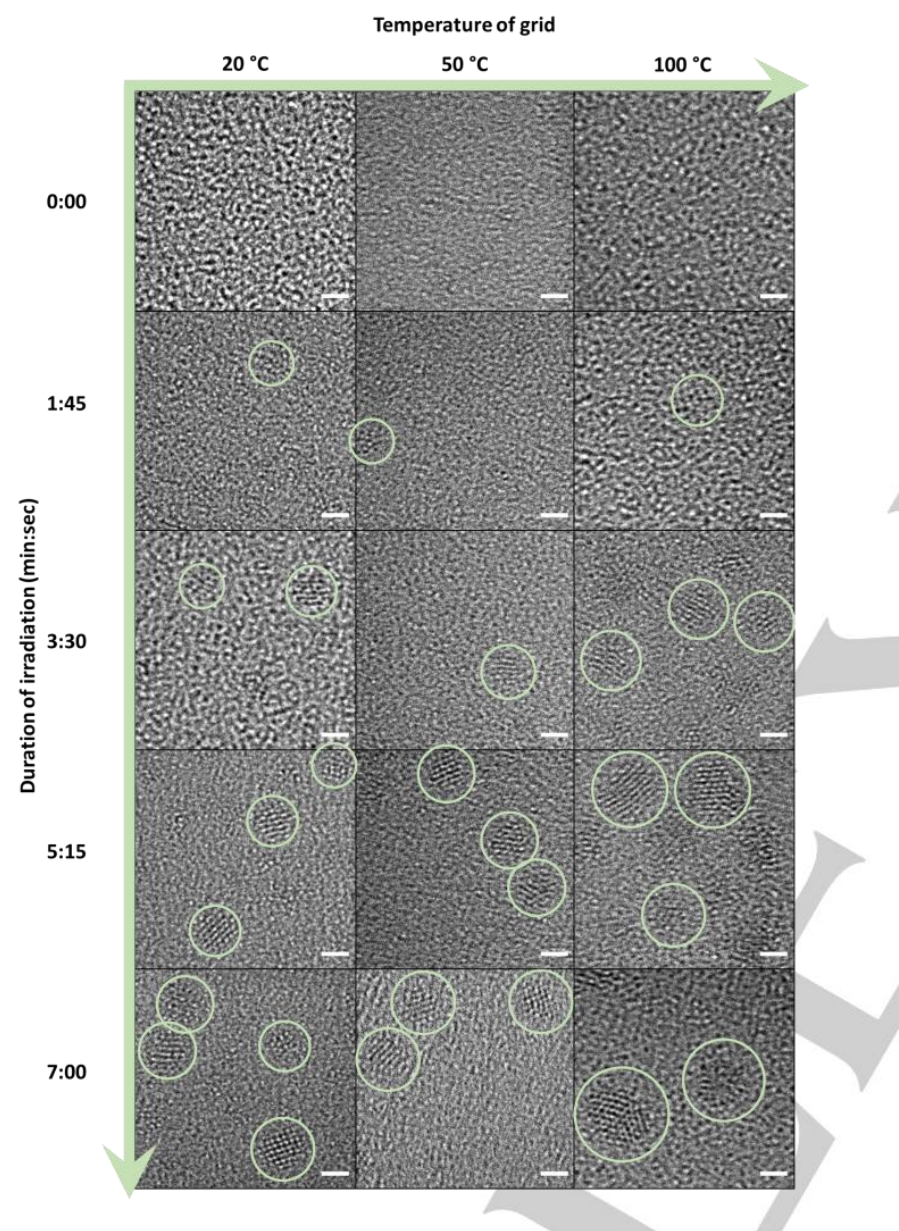

Figure 2. Growth of Os nanoclusters over 7 minutes at 20,50 , and $100{ }^{\circ} \mathrm{C}$ (scale bars $1 \mathrm{~nm}$ ).

The formation of clusters under the conditions of the ETEM was very fast and all the studies were conducted within a 7-min period. After less than $2 \mathrm{~min}$ of irradiation, clusters of ca. 0.85 $\mathrm{nm}$ of diameter at $20^{\circ} \mathrm{C}$ were already observed, while the average diameter reached ca. $0.92 \mathrm{~nm}$ at $50{ }^{\circ} \mathrm{C}$ and $0.96 \mathrm{~nm}$ at $100{ }^{\circ} \mathrm{C}$. Significantly, this in situ fabrication of Os clusters generates nanocrystals as early as after $5 \mathrm{~min}$ of irradiation. Indeed, the nanoclusters reached critical sizes and crystallised to yield nanocrystals of ca. $1.02 \mathrm{~nm}\left(20^{\circ} \mathrm{C}\right), 1.18 \mathrm{~nm}\left(50{ }^{\circ} \mathrm{C}\right)$, and $1.31 \mathrm{~nm}\left(100^{\circ} \mathrm{C}\right)$. Growth was imaged until 7 minutes (after which the surface started breaking down at $100^{\circ} \mathrm{C}$ ). Interestingly, the growth of the clusters was found to be linear over time for each of the three temperatures studied (Table 1; Figure 3;
Spreadsheet in the ESI: "diameter raw data" and "diameter analysis" tabs for each temperature).
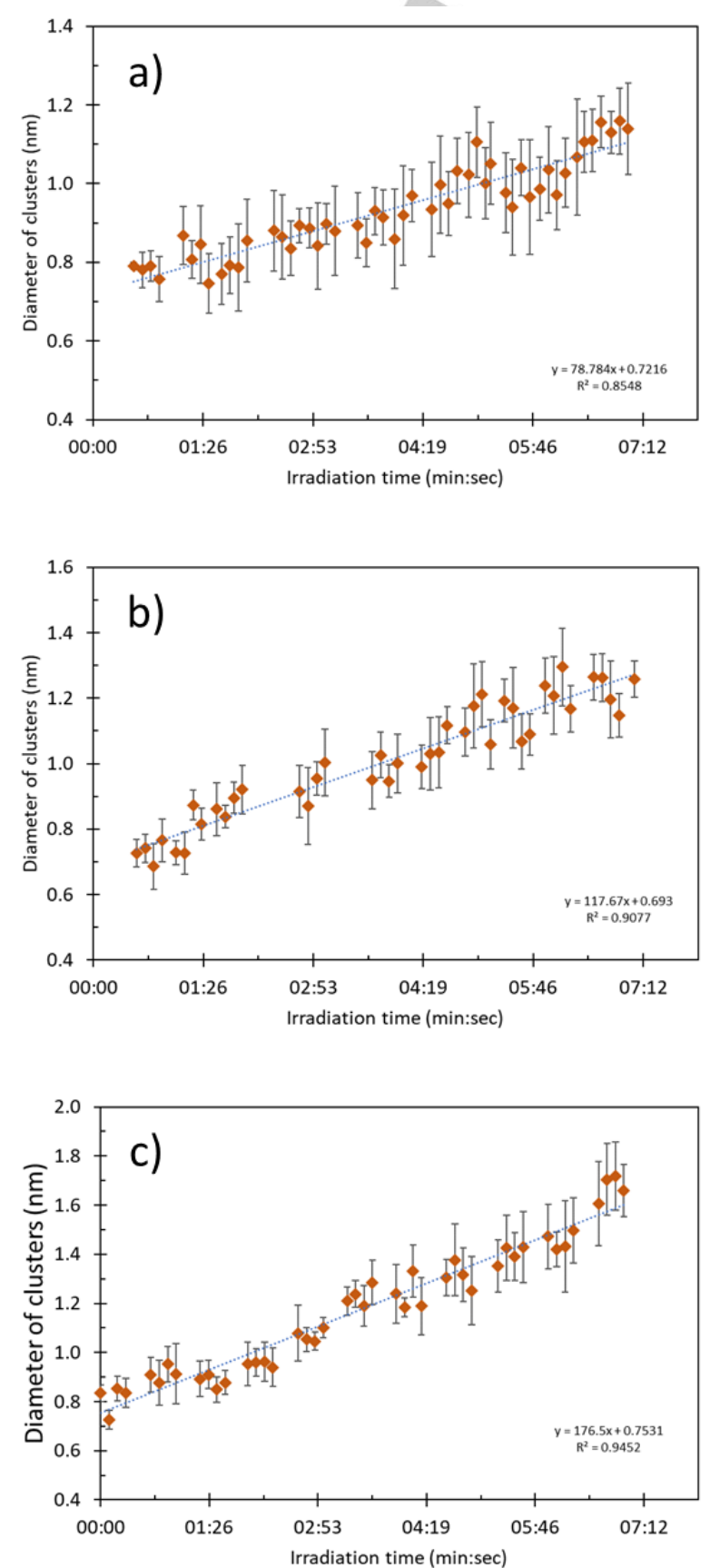

Figure 3. Diameter of the nanoclusters versus irradiation time at a) $20^{\circ} \mathrm{C}, \mathrm{b}$ ) $50{ }^{\circ} \mathrm{C}$, and c) $100{ }^{\circ} \mathrm{C}$. The error bars are the standard deviations from measurements obtained from as many clusters as possible (on average 10 per point, slightly less for early irradiation time).

A clear linear correlation between the nucleation rates and temperature $\left(78.8,117.7,176.5 \mathrm{pm} / \mathrm{min}\right.$ at $20,50,100{ }^{\circ} \mathrm{C}$, respectively) seems to emerge, although three points are not enough to prove such a linear correlation. Nonetheless, it is 
obvious that the nanocluster growth is directly influenced by the temperature of the grid. These results indicate that the effect of temperature on the nanocluster growth is of the same order of magnitude than the effect of underlying doping heteroatoms in the surface (26 times faster on Se-doped surfaces than on Sdoped surfaces; ${ }^{[96]} 2$ times faster on B-doped surface compared to B-free surface $\left.{ }^{[12]}\right)$. Temperature has also an effect of the same order of magnitude than the nature of the metal used to produce the nanoclusters. Indeed, we previously reported that the interactions of individual $\mathrm{Ru}, \mathrm{Os}, \mathrm{Au}$, and Ir atoms with the underlying surface have a direct but moderate impact on the rates of nucleation. ${ }^{[11]}$

Using the Arrhenius equation ("Activation energy" tab in the ESI spreadsheet for equations and calculations), the energy barrier of the thermally activated observed process was estimated to be $158.1 \mathrm{~J} / \mathrm{mol}$. The kinetic energy transferred from the e-beam to an osmium atom was also calculated, using the equation below ("Activation energy" tab in the ESI spreadsheet for equations and calculations) and was found to be $46.33 \mathrm{~J} / \mathrm{mol}$. This suggests that the energy received from the e-beam by an Os atom is not enough to trigger the knock-on atomic displacement of the Os atoms. However, the same calculations for carbon, boron, and sulfur atoms lead to energies greater than 158.1 $\mathrm{J} / \mathrm{mol}$ (815.2, 733.8, and $274.8 \mathrm{~J} / \mathrm{mol}$, respectively). This suggests that the kinetic energy of the electron beam is the main driving force for the observed dynamics of metal atoms in the TEM chamber, and that the motion of metal atoms is mainly triggered by the energy received by the surface rather than by the Os atoms.

$E_{\text {Tmax }}=\frac{2 \times m_{n} \times E \times\left(E+2 \times m_{e} \times c^{2}\right)}{\left(m_{n}+m_{e}\right)^{2}+2 \times m_{n} \times E}$

where $m_{n}=$ mass of atoms, $m_{e}=$ mass of electron, $E=$ energy of the e-beam, and $c=$ speed of light.

Table 1. Osmium nanocluster growth parameters.

\begin{tabular}{|c|c|c|c|}
\hline \multirow{2}{*}{$\begin{array}{l}\text { Temperature } \\
\left({ }^{\circ} \mathrm{C}\right)\end{array}$} & \multirow{2}{*}{$\begin{array}{c}\text { Growth rate } \\
\text { (pm/min) }\end{array}$} & \multicolumn{2}{|c|}{ Os-Os distance (nm) } \\
\hline & & Nanocluster & Bulk $^{[14]}$ \\
\hline 20 & 78.8 & $0.237 \pm 0.030$ & 0.2705 \\
\hline 50 & 117.7 & $0.241 \pm 0.030$ & 0.2705 \\
\hline 100 & 176.5 & $0.243 \pm 0.033$ & 0.2705 \\
\hline
\end{tabular}

Since temperature influences the rate of nanocrystallisation, we then investigated the effect of temperature on the structure of the nanocrystals (see ESI: "phase determination procedure" tab in the spreadsheet for a standard operating procedure, and the tabs "phase determination 20 C"; "phase determination $50 \mathrm{C}$ "; "phase determination $100 \mathrm{C}$ " for crystal structure determinations)

For each temperature, a number of crystals were analysed: first by generating the Fast Fourier transform (FFT) analysis from the TEM image; then the reciprocal lattice was compared with four known osmium-containing structures $(\mathrm{Os}(0)$ hexagonal; Os(0) cubic; Os(IV)O $\mathrm{O}_{2}$ tetragonal; Os(VIII)O $\mathrm{O}_{4}$ monoclinic) by using the software Diffraction-Workshop V2.2 (freeware to index TEM "dots" or "ring" diffraction patterns $\left.{ }^{[15]}\right)$. All crystals analysed matched with one or more of these four structures. At $20^{\circ} \mathrm{C}$, the osmium crystals do not seem to have a simple hexagonal structure (which is in accordance with our previous report ${ }^{[9 \mathrm{c}]}$ ). It should be noted that, because of the very small sizes of the nanocrystals (made up of less than 200 atoms in most cases), one such analysis can lead to the observation of more than one structure for the same crystal depending on the frame of observation (one crystal can fit with one structure at time $t$, and with another structure a few milliseconds later). In addition to the small diameter of the crystals $(<2 \mathrm{~nm})$, their $3 \mathrm{D}$ nature, as well as their rolling on the surface, also complicate the analysis of the phases. Furthermore, our attempt made at identifying the structures of the nanocrystals at the three temperatures $\left(20^{\circ} \mathrm{C}\right.$, $50{ }^{\circ} \mathrm{C}$, and $100{ }^{\circ} \mathrm{C}$ ) suggests that there is no obvious effect of the temperature on the crystallographic structures of the crystals since all four possible structures were observed at all temperatures (Figure 4).

In order to confirm/infirm the metallic nature of osmium under these conditions, a nanocrystal whose FFT analysis could not offer a clear phase determination was selected for further analysis (Figure 4). Indeed, comparison of the reciprocal lattice with osmium-containing structures (Os metal and Os oxides) leads to a possible phase corresponding to $\mathrm{Os}(\mathrm{IV}) \mathrm{O}_{2}$ tetragonal, $\mathrm{Os}(\mathrm{VIII}) \mathrm{O}_{4}$ monoclinic and $\mathrm{Os}(0)$ hexagonal structures. The observed bond lengths within this crystal made of ca. 60 apparent atoms were measured (Figure 4; ESI: "Distance M-M $100 \mathrm{C}$ " tab in the spreadsheet), along with the angles between the different rows of atoms. The average angle was found to be $62^{\circ}$ and the average distance between atoms to be $0.257 \mathrm{~nm}$. These experimental data fit well with osmium being mostly metallic, and they suggest that the $\operatorname{Os}(0)$ hexagonal is more likely to be the actual structure of this nanocrystal.

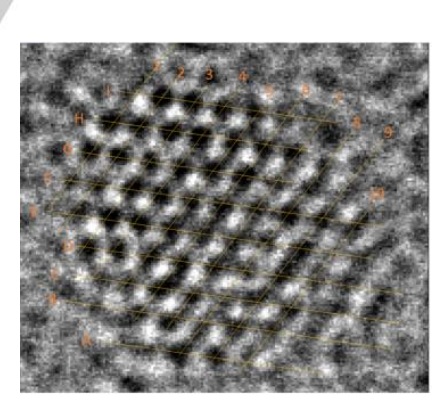

Average distance between consecutive Os atoms $(\mathrm{nm})$ Average angle: $62^{\circ}$

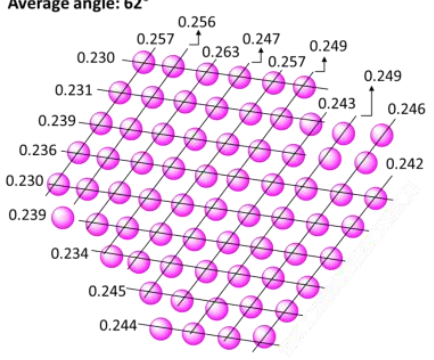

Figure 4. Os nanocrystal (imaged at $100^{\circ} \mathrm{C}$ ) with interatomic distances and angles within the nanocrystal.

The Os-Os bond lengths observed within this nanocrystal are also consistent with the calculated average Os-Os distance (Table 1; ESI: "Distance M-M for all T" tab in the spreadsheet). Indeed, the average Os-Os distances measured over clusters at $20^{\circ} \mathrm{C}, 50^{\circ} \mathrm{C}$, and $100^{\circ} \mathrm{C}$ were determined to be $0.237 \pm 0.030$, $0.241 \pm 0.030$, and $0.243 \pm 0.033 \mathrm{~nm}$, respectively. Interestingly, this study of average Os-Os distance over the three 
temperatures highlights that temperature does not seem to have an influence on the atomic arrangement within the nanocrystals. Furthermore, these Os-Os distances are close to those in crystals of bulk osmium metal $\left(0.27048 \mathrm{~nm}^{[14]}\right)$ and no change in metal-metal distance was observed during nanocluster growth.

In conclusions, using both capabilities of modern ETEMs (analytical tool and external force for the structural modification of nanomaterials), we generated experimental data on the nucleation and growth of nanocrystals of precious metal at three different temperatures. The growth rate was found to be dependent on the temperature (the nucleation being ca. 2.5 times faster at $100{ }^{\circ} \mathrm{C}$ than at $20^{\circ} \mathrm{C}$ ) in a seemingly linear fashion. Estimations of the energy barrier of the thermally activated observed process and of the kinetic energy transferred from the e-beam to both Os atoms and underlying surface (made of C, B, and S atoms) suggest that the motion of metal atoms is mainly triggered by the energy received by the surface rather than by the Os atoms. Importantly, no effect of the temperature on the crystal structures of the nanocrystals was observed, although the sizes of the crystals $(<2 \mathrm{~nm})$ and the very small number of atoms per crystal render a clear elucidation of the structures extremely difficult.

\section{Acknowledgements}

This project was supported by the Leverhulme Trust (Early Career Fellowship no. ECF-2013-414 to NPEB), the Royal Society (University Research Fellowship no. UF150295 to NPEB) and by the Academy of Medical Sciences/the Wellcome Trust/the Government Department of Business, Energy and Industrial Strategy/the British Heart Foundation Springboard Award [SBF003\1170 to NPEB]. We also thank the METSA national network (http://www.metsa.fr FR CNRS 3507) through its platform CLYM (http://www.clym.fr) and Dr. Thierry Epicier (University of Lyon, INSA - Lyon, France) for his help with the microscopy experiments on the ETEM and the stimulating discussions.

Keywords: Metal nanocrystals $•$ Nucleation $•$ Osmium • Aberration-corrected Transmission Electron Microscope •

[1] aD.-W. Lim, J. W. Yoon, K. Y. Ryu, M. P. Suh, Angew. Chem. Int. Ed. 2012, 51, 9814-9817; bJ.-S. Lee, M. V. Kovalenko, J. Huang, D. S Chung, D. V. Talapin, Nat Nano 2011, 6, 348-352; cY. T. Zhu, X. Liao, Nat. Mater. 2004, 3, 351-352; dP. Wu, X.-P. Yan, Chem. Soc. Rev. 2013, 42, 5489-5521; eM. Cargnello, V. V. T. Doan-Nguyen, T. R Gordon, R. E. Diaz, E. A. Stach, R. J. Gorte, P. Fornasiero, C. B. Murray, Science 2013, 341, 771-773; fY. Yamada, C.-K. Tsung, W Huang, Z. Huo, S. E. Habas, T. Soejima, C. E. Aliaga, G. A. Somorjai, P. Yang, Nat. Chem. 2011, 3, 372-376; gD. Jariwala, V. K. Sangwan, L. J. Lauhon, T. J. Marks, M. C. Hersam, Chem. Soc. Rev. 2013, 42 2824-2860; hE.-K. Lim, T. Kim, S. Paik, S. Haam, Y.-M. Huh, K. Lee, Chem. Rev. 2014; iJ. Yao, M. Yang, Y. Duan, Chem. Rev. 2014, 114 6130-6178; jJ. A. Hubbell, A. Chilkoti, Science 2012, 337, 303-305; kB Cornelio, G. A. Rance, M. Laronze-Cochard, A. Fontana, J. Sapi, A. N Khlobystov, J. Mater. Chem. A 2013, 1, 8737-8744; IS. T. Skowron, T.
W. Chamberlain, J. Biskupek, U. Kaiser, E. Besley, A. N. Khlobystov, Acc. Chem. Res. 2017, 50, 1797-1807.

[2] J. Jortner, C. N. R. Rao, Pure Appl. Chem. 2002, 74, 1491-1506.

[3] aG. Madras, B. J. McCoy, Chem. Eng. Sci. 2004, 59, 2753-2765; bS. A Miners, M. W. Fay, M. Baldoni, E. Besley, A. N. Khlobystov, G. A. Rance, The Journal of Physical Chemistry C 2019, 123, 6294-6302; cM. A. Astle, G. A. Rance, H. J. Loughlin, T. D. Peters, A. N. Khlobystov, Adv. Funct. Mater. 2019, 29, 1808092; dK. Cao, T. Zoberbier, J Biskupek, A. Botos, R. L. McSweeney, A. Kurtoglu, C. T. Stoppiello, A. V. Markevich, E. Besley, T. W. Chamberlain, U. Kaiser, A. N. Khlobystov, Nature Communications 2018, 9, 3382.

[4] J. Lee, W. Zhou, S. J. Pennycook, J.-C. Idrobo, S. T. Pantelides, Nat. Commun. 2013, 4, 1650

[5] J. A. Rodríguez-Manzo, O. Cretu, F. Banhart, ACS Nano 2010, 4 3422-3428.

[6] J. Kotakoski, C. Mangler, J. C. Meyer, Nat Commun 2014, 5, 4991.

[7] aS. R. Plant, L. Cao, R. E. Palmer, J. Am. Chem. Soc. 2014, 136, 7559-7562; bS. R. Plant, L. Cao, F. Yin, Z. W. Wang, R. E. Palmer, Nanoscale 2014, 6, 1258-1263; cY. Han, D. S. He, Z. Y. Li, J. Nanopart. Res. 2013, 15, 1-7; dA. La Torre, M. del Carmen Gimenez-Lopez, M. W. Fay, C. H. Lucas, P. D. Brown, A. N. Khlobystov, Small 2015, 11, 27562761.

[8] T. W. Chamberlain, J. Biskupek, S. T. Skowron, A. V. Markevich, S. Kurasch, O. Reimer, K. E. Walker, G. A. Rance, X. Feng, K. Müllen, A Turchanin, M. A. Lebedeva, A. G. Majouga, V. G. Nenajdenko, U Kaiser, E. Besley, A. N. Khlobystov, ACS Nano 2017, 11, 2509-2520.

[9] aA. Pitto-Barry, L. M. A. Perdigao, M. Walker, J. Lawrence, G Costantini, P. J. Sadler, N. P. E. Barry, Dalton Trans. 2015, 44, 2030820311; bN. P. E. Barry, A. Pitto-Barry, J. Tran, S. E. F. Spencer, A. M. Johansen, A. M. Sanchez, A. P. Dove, R. K. O'Reilly, R. J. Deeth, R. Beanland, P. J. Sadler, Chem. Mater. 2015, 27, 5100-5105; cN. P. E Barry, A. Pitto-Barry, A. M. Sanchez, A. P. Dove, R. J. Procter, J. J. Soldevila-Barreda, N. Kirby, I. Hands-Portman, C. J. Smith, R. K O'Reilly, R. Beanland, P. J. Sadler, Nat. Commun. 2014, 5, 3851.

[10] aX. Qi, Y. Huang, M. Klapper, F. Boey, W. Huang, S. D. Feyter, K. Müllen, H. Zhang, J. Phys. Chem. C 2010, 114, 13465-13470; bJ.-U Kim, S.-H. Cha, K. Shin, J. Y. Jho, J.-C. Lee, J. Am. Chem. Soc. 2005 127, 9962-9963; cA. H. Latham, M. E. Williams, Langmuir 2008, 24 14195-14202; dA. H. Latham, M. J. Wilson, P. Schiffer, M. E. Williams J. Am. Chem. Soc. 2006, 128, 12632-12633; eC. W. Huang, C. L. Hsin, C. W. Wang, F. H. Chu, C. Y. Kao, J. Y. Chen, Y. T. Huang, K. C. Lu, W. W. Wu, L. Chen, J. Nanoscale 2012, 4, 4702-4706; fJ. W. Liu, J. E. Xu, Y. Ni, F. J. Fan, C. L. Zhang, S. H. Yu, ACS Nano 2012, 6, 4500 4507; gZ. W. Wang, R. E. Palmer, Phys. Rev. Lett. 2012, 108, 245502.

[11] A. Pitto-Barry, P. J. Sadler, N. P. E. Barry, Chem. Commun. 2016, 52, 3895-3898.

[12] A. Pitto-Barry, N. P. E. Barry, Chem. Commun. 2019, 55, 6038-6041.

[13] N. P. E. Barry, A. Pitto-Barry, I. Romero-Canelon, J. Tran, J. J. Soldevila-Barreda, I. Hands-Portman, C. J. Smith, N. Kirby, A. P. Dove R. K. O'Reilly, P. J. Sadler, Faraday Discuss. 2014, 175, 229-240.

[14] J. W. Arblaster, Platinum Metals Rev. 1989, 33, 14-16.

[15] https://www.clym.fr/fr/node/131. 


\section{Entry for the Table of Contents}

\section{COMMUNICATION}

Direct observation of the effect of temperature on the nucleation and growth of metal nanocrystals

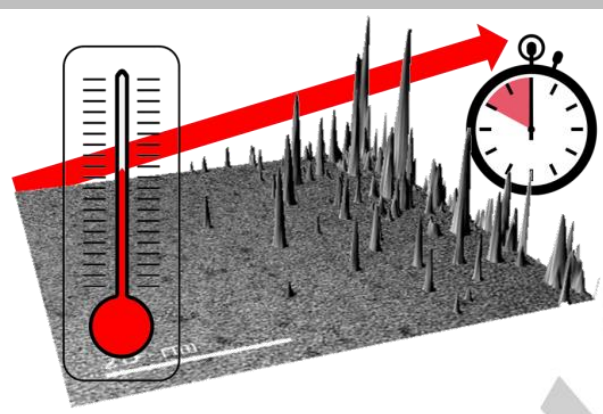

Anaïs Pitto-Barry* and Nicolas P. E. Barry*

Page No. - Page No.

Effect of temperature on the nucleation and growth of precious metal nanocrystals 